\title{
Energetics and Kinetics of S-State Transitions Monitored by Delayed Chlorophyll Fluorescence
}

\author{
Ivelina Zaharieva* and Holger Dau* \\ Department of Physics, Freie Universität Berlin, Berlin, Germany
}

Understanding energetic and kinetic parameters of intermediates formed in the course of the reaction cycle (S-state cycle) of photosynthetic water oxidation is of high interest and could support the rationale designs of artificial systems for solar fuels. We use time-resolved measurements of the delayed chlorophyll fluorescence to estimate rate constants, activation energies, free energy differences, and to discriminate between the enthalpic and the entropic contributions to the decrease of the Gibbs free energy of the individual transitions. Using a joint-fit simulation approach, kinetic parameters

OPEN ACCESS

Edited by:

Marcin Rapacz,

University of Agriculture of Krakow.

Poland

Reviewed by:

Roman Dědic,

Charles University, Czechia David Kramer

Michigan State University,

United States

*Correspondence:

Ivelina Zaharieva

ivelina.zaharieva@fu-berlin.de

Holger Dau

holger.dau@fu-berlin.de

Specialty section:

This article was submitted to

Plant Abiotic Stress,

a section of the journal

Frontiers in Plant Science

Received: 03 January 2019

Accepted: 13 March 2019

Published: 29 March 2019

Citation:

Zaharieva I and Dau H (2019) Energetics and Kinetics of S-State Transitions Monitored by Delayed

Chlorophyll Fluorescence.

Front. Plant Sci. 10:386.

doi: 10.3389/fpls.2019.00386 are determined for the reaction intermediates in the $S$-state transitions in buffers with different $\mathrm{pH}$ in $\mathrm{H}_{2} \mathrm{O}$ and in $\mathrm{D}_{2} \mathrm{O}$.

Keywords: delayed chlorophyll fluorescence, protonation dynamics, S-state cycle intermediates, thermodynamics, activation energies

\section{INTRODUCTION}

Water oxidation is one of the chemical reactions with largest impact on the planet as it is responsible for the production of the atmospheric $\mathrm{O}_{2}$. Recently understanding of the mechanism of water oxidation became of increasing importance, as this reaction is the ultimate source of protons and electrons to be used in to synthesize renewable fuels. Water oxidation takes place in thylakoid membranes of higher plants, algae and cyanobacteria (Ort and Yocum, 1996; Blankenship, 2002; Nelson and Junge, 2015), where it is catalyzed by a manganese-calcium complex embedded in the highly conserved protein environment of Photosystem II protein, PSII (Nelson and Yocum, 2006; Williamson et al., 2011; Pagliano et al., 2013). Breakthroughs in protein crystallography revealed location and structure of the oxygen-evolving complex (OEC), which consists of $\mathrm{Mn}_{4} \mathrm{Ca}(\mu-\mathrm{O})_{5}$ cluster surrounded by specific sidechains and functionally important water molecules (Zouni et al., 2001; Ferreira et al., 2004; Umena et al., 2011; Suga et al., 2015; Kern et al., 2018). In spite of recent progress in crystallographic characterization (e.g., Kern et al., 2018) and advanced biophysical investigation of PSII function (e.g., Klauss et al., 2012), the characteristics and mechanistic role of intermediates in the reaction cycle of water oxidation is insufficiently understood. Resolving and understanding transiently formed intermediates of the PSII water oxidation cycle could pave the road toward a complete atomistic picture of the mechanism of photosynthetic water oxidation.

When exposing dark-adapted photosynthetic organisms (or isolated photosystems) to a sequence of saturating flashes of visible light, a characteristic patter of period-of-four oscillations is observed for the flash-number dependence of $\mathrm{O}_{2}$-formation (Joliot et al., 1969). This characteristic flash pattern has been explained by Kok et al. (1970) by the basic $S$-state cycle model describing the accumulation of four "positive charges" before onset of dioxygen formation and assuming 5 intermediate states labeled $S_{0}$ to $S_{4}$ (Kok et al., 1970). Later it has been realized that not only 
electrons are removed from the $\mathrm{Mn}_{4} \mathrm{CaO}_{5}$ cluster (by the Tyr 161 of the D1 protein, Tyr161, YZ, Faller et al., 2001, 2003) but also protons are removed from the OEC (Junge et al., 2002) so that the extent of charge accumulation depends on the temporal sequence of electron and proton removal steps. Consequently, Kok's basic reaction cycle does not describe the accumulation of four of four positive charges, but of four oxidizing equivalents before the onset of the O-O bond formation. In 2007 an extended S-state cycle was proposed that explicitly takes into account electron and proton removal steps, where alternating electron-proton removal prevents charge accumulation and a prohibitive increase in the redox potential of the active-site metal complex, as illustrated in Figure 1 (Dau and Haumann, 2007a,b). Clear experimental evidences for $S$-state cycle intermediates in the extended S-state cycle is available only for some of the reactions (Haumann et al., 2005; Gerencser and Dau, 2010; Klauss et al., 2012; Zaharieva et al., 2016). For all transiently formed intermediates, their energetic, kinetic and structural properties are insufficiently understood. Addressing the knowledge gaps regarding the reaction kinetics, motivates our study.

Fluorescence measurements are highly informative method to address the function of Photosystem II, as they can provide a variety of information about the photosynthetic apparatus in its native state (Kalaji et al., 2014, 2017). Typically, the variability of the chlorophyll (Chl) fluorescence yield is recorded after sudden application of an actinic light, in the time range form microseconds to tens of minutes (fluorescence induction curves). However, often high complexity hinders quantitative interpretation in terms of reaction kinetics of specific molecular events seriously. This is even more the case for the delayed chlorophyll fluorescence $(D F$, recombination fluorescence) (Goltsev et al., 2009). Thus, we use laser-flash excitation for investigation of the events in PSII. Unlike induction kinetics, the decays measured after nanosecond laser-flash illumination of dark-adapted samples provide better-defined starting conditions and a more direct access to information about the reactions taking place in PSII at various times after flash-excitation.

Light excitation of dark adapted PSII results in the formation of excited singlet states of $\mathrm{Chl}$ followed by rapid excitation energy transfer and equilibration among the antenna chlorophylls of PSII (rapid exciton equilibration) (Schatz et al., 1988; Dau and Sauer, 1996). Excitation of the chloropyll(s) denoted as P680 results in formation of $\mathrm{P} 680^{*}$, which initiates the primary charge separation and leads to reduction of a specific pheophytin (Phe) and formation of $\mathrm{P}_{680}{ }^{+}$, the oxidized primary donor (Figure 1). The pheophytin is reoxidized by electron transfer to the primary quinone acceptor, $\mathrm{Q}_{\mathrm{A}}$. The $\mathrm{P}_{680}{ }^{+}$states are "quenched" mostly by a donation of an electron from the primary electron donor, $\mathrm{Y}_{\mathrm{Z}}$, to $\mathrm{P}_{680}{ }^{+}$within less than $1 \mu \mathrm{s}$ after the Laser flash. The $\mathrm{Y}_{\mathrm{Z}}$ oxidation is followed by an oxidation of the $\mathrm{Mn}_{4} \mathrm{CaO}_{5}$ cluster coupled by structural rearrangements and proton-removal steps. $\mathrm{Q}_{\mathrm{A}}{ }^{-}$decays are mostly due to electron transfer to $\mathrm{Q}_{B}$ at the acceptor side, a clearly slower process with a half-time of several milliseconds.

The chlorophyll fluorescence intensity is proportional to the fraction of excited antennae-chlorophyll molecules $\left[\mathrm{Chl}^{*}\right]$ and to the probability to decay via fluorescence emission. For the chlorophyll fluorescence (prompt fluorescence, PF), which is measured as a fluorescence intensity during illumination, there is a clear relation between the probability for charge separation (formation of $\mathrm{P} 680^{+} \mathrm{Q}_{\mathrm{A}}{ }^{-}$radical pair) and the $\mathrm{PF}$ intensity, manifested in increase of PF with accumulation of reduced $\mathrm{Q}_{\mathrm{A}}{ }^{-}$ and vise versa (Dau, 1994). When measurements are performed in darkness, and the time interval between the light excitation and detection is in the microsecond or millisecond domain (and thus clearly longer than the lifetime of excited chlorophyll molecules), $\mathrm{PF}$ is not detectable but a weak fluorescence emission (delayed fluorescence, $D F$ ) that relates to repopulation of the $\mathrm{Chl}^{*}$ state by recombination of charge-separated radical pairs can be recorded. The actual population of the $\mathrm{Chl}^{*}$-state in this case is determined by the free-energy difference between the excited-antenna state and the radical-pair state $\left[\mathrm{Yz}^{+} \mathrm{Q}_{\mathrm{A}}{ }^{-}\right]$reached at the respective time (Buchta et al., 2007; Goltsev et al., 2009):

$$
D F(t) \sim\left[\operatorname{chl} l^{*}\right]=\left[\operatorname{Tyr}_{z}^{\bullet+} Q_{A}^{-}\right](t) e^{\Delta G_{A \bullet R P} / k_{B} T} \quad \text { Eq. } 1.1
$$

Where $\Delta \mathrm{G}_{A^{*} R P}$ is the difference in the Gibbs free energy $(\Delta G<0)$ between the excited antenna $\left(\mathrm{Chl}^{*}\right)$ and the PSII radical-pair state $\left[\mathrm{Yz}^{\bullet}{ }^{+} \mathrm{Q}_{\mathrm{A}}{ }^{-}\right], \mathrm{k}_{\mathrm{B}}$ is the Boltzmann constant, and $\mathrm{T}$ is the absolute temperature in Kelvin.

The rapid decrease in the $D F$ fluorescence measured in the dark after a flash-excitation, is due to the decrease in the concentration of $\left[\mathrm{Yz}^{\bullet}{ }^{+} \mathrm{Q}_{\mathrm{A}}{ }^{-}\right]$states by (i) electron transfer from $\mathrm{Q}_{\mathrm{A}}$ to $\mathrm{Q}_{\mathrm{B}}$ (reoxidation of the reduced acceptor $\left(\mathrm{Q}_{\mathrm{A}}{ }^{-}\right)$, (ii) reduction of $\mathrm{Y}_{Z}{ }^{\bullet+}$ by electron transfer from the $\mathrm{Mn}_{4} \mathrm{CaO}_{5}$ complex and (iii) charge recombination between $\mathrm{Y}_{Z}{ }^{\bullet+}$ and $\mathrm{Q}_{A}{ }^{-}$, where the latter is a competing process that depends on the concentrations of the $\left[\mathrm{Yz}^{\bullet+} \mathrm{Q}_{\mathrm{A}}{ }^{-}\right]$state and thus also on the first two reactions (Goltsev et al., 2009). Also, completion of electron transfer to $\mathrm{Y}_{Z}{ }^{\bullet+}$ does not eliminate $D F$ emission completely, because a fraction of PSII will still harbor $\mathrm{Y}_{\mathrm{Z}}$, according to the Gibbs free energy difference of this electron transfer step (Figure 1). This means that after the time required for an electron transfer from $\mathrm{Mn}_{4} \mathrm{CaO}_{5}$ cluster to $\mathrm{Y}_{\mathrm{Z}}{ }^{\bullet+}$, there is a fraction of reaction centers where $\mathrm{Y}_{\mathrm{Z}}{ }^{\bullet+}$ is still present. Moreover, $D F(\mathrm{t})$ decreases because proton movements or structural changes stabilize the radical pair. The way all these processes affect the $D F$ signal can be generalized: $D F(\mathrm{t})$ reflects the total Gibbs free energy differences (toward the excited antenna state) of any state formed in the reaction sequence. If we can identify the decrease in the $D F$ decay to a transition between state-A and state- $B$ in the $D F$ decay (e.g., by assignment of an exponential decay), the corresponding $\triangle \mathrm{G}_{A B}$ can be calculated from the $D F$ level of state- $\mathrm{A}, D F(\mathrm{~A})$, and the (lower) $D F$ level of state- $\mathrm{B}, D F(\mathrm{~B})$, according to:

$$
\Delta G_{A B}=-k_{B} T \ln \frac{D F(A)}{D F(B)} \quad E q .1 .2
$$

Here $D F(A)$ corresponds to the $D F$ level before onset of the $\mathrm{A} \rightarrow \mathrm{B}$ transition and $D F(\mathrm{~B})$ corresponds the $D F$ level after its completion. Using this rationale, analysis of the $D F$ signal can provide number not only for the time constants 


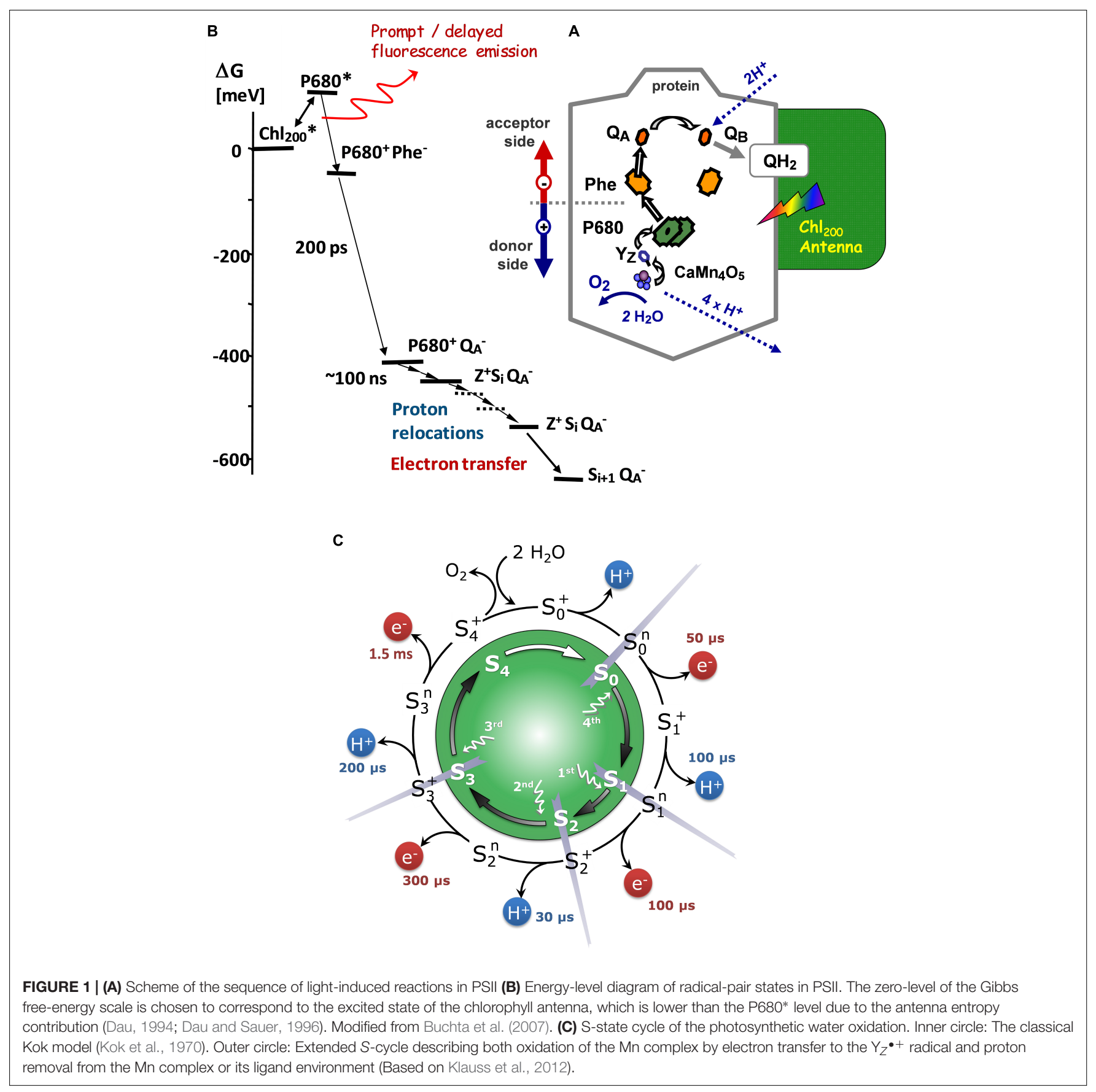

of the transition from state-A to state-B, but also for the corresponding $\triangle \mathrm{G}_{A B}$.

In Buchta et al. (2007) a rationale was developed to correct $D F$ decays measured after ns-Laser flash excitation for the contribution of $\mathrm{QA}^{-}$reoxidation by combined measurements of $D F$ decays and prompt fluorescence decay under similar experimental conditions. Applying this method, the DF decay can be used to obtain information mostly about the processes at the donor side of PSII, related to the proton and electron transfer at the $\mathrm{Mn}_{4} \mathrm{CaO}_{5}$ cluster. The bottleneck in using the potentially highly informative $D F$ analysis is the complexity of the $D F$ decays, where the individual kinetic components contributing to the total DF decay are difficult to resolve.

In the present work, we analyze decays of the DF fluorescence of PSII extending from $10 \mu \mathrm{s}$ to $10 \mathrm{~ms}$. Using a multiexponential model, a high-quality fit typically requires minimally four exponential components, resulting in nine independent fit parameters. Typically, parameter correlations will render several of these nine parameters uncertain. Therefore, in past studies, mostly a three-exponential model was employed (Haumann et al., 2005; Buchta et al., 2007; Zaharieva et al., 2011) for simulation of the third-flash decays. Then the resulting fit 
parameters were used to calculate three potentially informative figures: the time-constant of $\mathrm{O}_{2}$ formation, the total Gibbs free energy of the proton removal steps, and a mean timeconstant for all proton removal steps. These three figures are largely insensitive to fit-parameter correlations, but especially the inability to resolve the time-constants of the proton removal step individually represents a serious drawback. In our study, a jointfit simulation approach that allows to overcome, at least partially, this limitation is presented.

\section{MATERIALS AND METHODS}

\section{Sample Preparation}

Highly active PSII membrane particles were prepared from market spinach according to Schiller and Dau (2000). The rate of $\mathrm{O}_{2}$ evolution under continuous illumination with white light at $28^{\circ} \mathrm{C}$ was about $1000-1400 \mu$ mol.mg chl ${ }^{-1} \cdot \mathrm{h}^{-1}$ and the Chl $a / \mathrm{Chl} b$ ratio was about 2.5 . After preparation the membrane particles were stored at $-80^{\circ} \mathrm{C}$ in buffer A: $25 \mathrm{mM}$ MES (2morpholinoethanesulfonic acid, 99.5\%, Merck, $\left.\mathrm{pK}_{a}=6.15\right)$, $10 \mathrm{mM} \mathrm{NaCl}$ ( $\geq 99.8 \%$, Roth), $5 \mathrm{mM} \mathrm{MgCl} 2$ ( $\geq 99 \%$, Roth), $5 \mathrm{mM} \mathrm{CaCl}_{2}$ ( $\geq 98 \%$, Roth), $1 \mathrm{M}$ glycine-betaine (betainemonohydrate, Alfa Aesar, 99\%), pH 6.4 (adjusted with $\mathrm{NaOH}$ ). The PSII particles were stored in Ependorff vials at a chlorophyll concentration of $2-3 \mathrm{mg} / \mathrm{ml}$.

Before measurements, $1 \mathrm{ml}$ PSII suspension (chlorophyll concentration of about $2 \mathrm{mg} \mathrm{chl} . \mathrm{ml}^{-1}$ ) was thawed on ice (for $60 \mathrm{~min}$, in the dark) and gently resuspended in $2-3 \mathrm{ml}$ buffer $\mathrm{A}$ using a soft brush. The volume was adjusted to $40 \mathrm{ml}$ with Buffer $\mathrm{A}$ and the protein sample centrifuged $12 \mathrm{~min}$ at $48000 \mathrm{~g}$ in order to remove residuals of starch and free chlorophyll. The pellet was resuspended in 3-4 milliliters of buffer B (25 mM MES, $25 \mathrm{mM}$ MOPS (3-morpholinopropanesulfonic acid, 99,5\%, AppliChem, $\left.\mathrm{pK}_{a}=4.0\right), 25 \mathrm{mM}$ HEPPS (4-(2-hydroxyethyl) piperazine-1propanesulfonic acid, 98\%, AppliChem, $\mathrm{pK}_{a}=8.0$ ), $10 \mathrm{mM} \mathrm{NaCl}$, $5 \mathrm{mM} \mathrm{MgCl}_{2}, 5 \mathrm{mM} \mathrm{CaCl}_{2}, 1 \mathrm{M}$ glycine-betaine, $\mathrm{pH}$ adjusted to 6.4 or 5.2 with concentrated solutions of $\mathrm{NaCl}$ or $\mathrm{HCl}$ ). To account the different sensitivity of the standard $\mathrm{pH}$ electrode to protons and deuterons, a correction to the value read from the $\mathrm{pH}$ meter in $\mathrm{D}_{2} \mathrm{O}$ buffer ( $\mathrm{pH}^{\prime}$ ) was done according to $\mathrm{pD}=\mathrm{pH}^{\prime}+0.4$ (Glasoe and Long, 1960).

Chlorophyll concentration was determined in 80\% acetonewater mixture according to Lichtenthaler (1987) using Cary 50 Conc UV-Vis Spectrophotometer, Varian. The chlorophyll concentration was adjusted with Buffer B to $100 \mu \mathrm{g} / \mathrm{ml}$ and the diluted suspension stored in ice in dark during the $D F$ measurements, but no longer that $1 \mathrm{~h}$. One minute before the measurements directly in a cuvette the PSII suspension was mixed with Buffer B and DCBQ [2,6-dichloro-1,4benzoquinone, Aldrich, 98\%, 1\% DCBQ in water-DMSO mixture (dimethylsulfoxid, Merck)] to a final concentration of $10 \mu \mathrm{g}$ $\mathrm{chl} / \mathrm{ml}, 20 \mu \mathrm{mol}$ DCBQ and final volume of $1.5 \mathrm{ml}$.

\section{Time-Resolved Measurements of Prompt Chl Fluorescence}

The yield of the prompt fluorescence for excitation with a sequence of laser-Continuum Minilite II (532 nm, FWHM of
$5 \mathrm{~ns}, 2 \mathrm{~mJ} / \mathrm{cm}^{2}, 700 \mathrm{~ms}$ interval between flashes) was detected at various times in a pump-probe experiment by means of commercially available instrument (FL 3000, Photon Systems Instruments) and used for correction of the $D F$ decays in order to remove the contribution of the $\mathrm{QA}^{-}$decay due to $\mathrm{QA}^{-}$ reoxidation by $\mathrm{Q}_{\mathrm{B}}$ (acceptor side contribution) as described elsewhere (Buchta et al., 2007).

\section{Time-Resolved Delayed Fluorescence Measurements}

Delayed fluorescence measurements were performed by an inhouse setup as described in Grabolle and Dau (2005). For the measurements, a polystyrene (PS) cuvette (BRAND, 340$900 \mathrm{~nm}$, length path $10 \mathrm{~mm}$ ) with four clear sides was placed in cuvette holder made from copper and facilitating temperature control by a flow-through system. By means of a water bath thermostat (Circulator DC50, bath K40, HAAKE), the temperature of the PSII suspension was set at a fixed value in the range between 0 and $30^{\circ} \mathrm{C}$, with an estimated accuracy of $0.2^{\circ} \mathrm{C}$. The dark-adapted samples were excited by a saturating Laser flash of $2 \mathrm{~mJ} / \mathrm{cm}^{2}$ (Continuum Minilite II, $532 \mathrm{~nm}$, FWHM of $5 \mathrm{~ns}$, time between flashes of $0.7 \mathrm{~s}$ ). The delayed fluorescence signal was recorded by a gated photomultiplier (Hamamatsu R2066; PMT Gated Socket Assembly C1392-55; anode voltage, $1000 \mathrm{~V}$; anode resistor, $2.2 \mathrm{kOhm}$; gating voltage, $240 \mathrm{~V}$ applied from $7 \mu \mathrm{s}$ before to $3 \mu \mathrm{s}$ after the Laser flash). Scattered Laser light was suppressed by a combination of two long-pass filters (LINOS Photonics, DT-red and DTmagenta with cut-off wavelength 600 and $632 \mathrm{~nm}$, respectively). After amplification (Tektronix AM502, bandwidth of $300 \mathrm{kHz}$ ), the signal was sampled at $1 \mathrm{MHz}$ by a 12-bit PC-card (ADLINK, PCI 9812). At least three repetitions were done for each measurement and averaged after applying the corrections described below. The reproducibility of the data is illustrated in the Supplementary Figure S10.

\section{Data Analysis}

The DF decays obtained after each Laser flash were corrected for an artifact of the detector system as described elsewhere (Grabolle and Dau, 2005). The artifact predominantly was due to excitation of delayed fluorescence in the glass or cathode material of the photomultiplier, by the strong prompt fluorescence of the PSII particles as well as by scattered light of the excitation flash. Additionally, the $D F$ signal was corrected for the contribution from the $\left[\mathrm{Q}_{A}{ }^{-}\right]$decay (Buchta et al., 2007).

The obtained $D F$ decay curve was simulated as a sum of four exponential decays plus an offset (Eq. 2.1).

$$
F(t)=\sum_{i=1}^{4} a_{i} \exp \left(-t / \tau_{i}\right)+c \quad \text { Eq. } 2.1
$$

The parameters $a_{i}, \tau_{i}$, and $c$ (9 parameters in total for each $D F$ decay) were determined by minimization of the error sum. For curve-fitting and in all figures, we averaged the delayed fluorescence decays "logarithmically" such that a linear spacing 


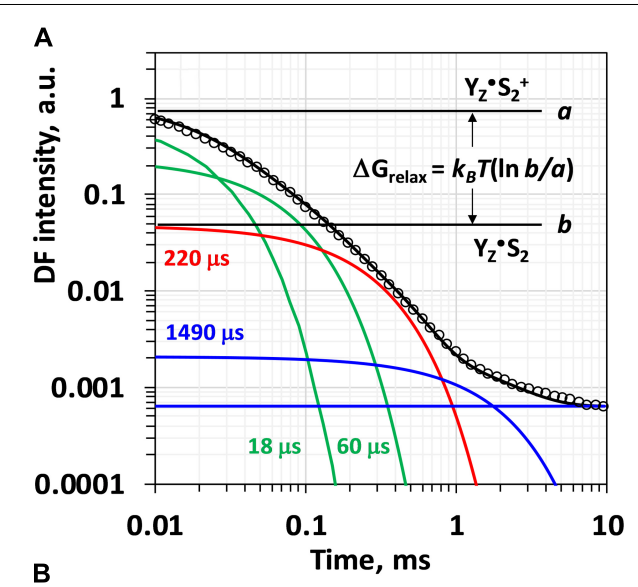

B

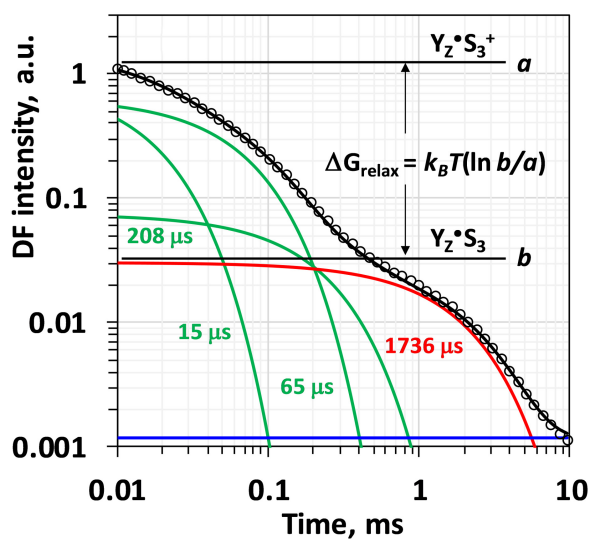

FIGURE 2 | Simulation of the delayed fluorescence decay measured after second (A) and after third (B) ns-flash excitation of dark adapted PSII particles at $20^{\circ} \mathrm{C}$ and $\mathrm{pH}$ 6.4. Experimental points are shown with circles, simulated curves according to Eq. 2.1 within the joint fit simulation approach as solid black lines. The individual components are also shown: green lines and corresponding time constants represent the components used to simulate the multiphasic process of intermediate formation associated with $\mathrm{H}^{+}$release step, red lines represent the electron transfer step $\left(\mathrm{Mn}_{4} \mathrm{CaO}_{5} \rightarrow \mathrm{Y}_{Z}{ }^{\bullet+}\right)$ and blue lines model the slow reactions presumably not related to processes at the donor side of PSII. The change in the Gibbs free energy, $\Delta G$, is calculated according the equations 3.3-3.6 from the ratio of the levels before and after the reaction of interest (the case shown in the plot is for the total free energy change, $\Delta G_{\text {relax }}$, for the reactions that take place before the electron transfer step).

of the points on a logarithmic time axis was achieved. Then the error sum was calculated according to:

$$
\varepsilon^{2}=\sum_{N}\left(\log \frac{F_{\text {sim }}}{F_{\text {sim }}}\right)^{2}
$$

In order to avoid overparametrization, a joint simulation approach was developed as detailed in the "Results" section. Simulations were done by in-house software developed by Dr. P. Chernev (Chernev, 2007) using Object Pascal programming language and Simulated Annealing minimization algorithm (van Laarhoven and Aarts, 1987).

\section{RESULTS}

\section{Simulation Approach}

Visual inspection of the $D F$ decays recorded after the third flash reveals a biphasic behavior (Figure 2). The first phase extends over the first $0.3 \mathrm{~ms}$ and has been associated with intermediate formation by proton removal from the oxygen evolving complex by a deprotonation step during the $S_{3} \rightarrow$ $S_{0}$ transition (Haumann et al., 2005; Buchta et al., 2007). The second step with a halftime of about $1.7 \mathrm{~ms}$ is assignable to the electron transfer step associated with dioxygen formation (Klauss et al., 2012). Simulation with a sum of two exponential decays only, however, has been proven to be clearly unsatisfactory (Buchta et al., 2007). Excellent agreement between experimental data and simulated curve can be achieved only by fourexponential fit (Supplementary Figure S1) suggesting that the deprotonation step is not a simple single-phase process, but at least three, presumably sequential reaction steps are involved (Buchta et al., 2007). Using four exponentials according to Eq. 2.1 would require 9 free parameters ( 5 amplitudes and 4 lifetimes) to simulate a single $D F$ decay and the fit will be strongly undetermined. This would hamper the determination of the individual rate constants as well as the calculation of the free-energy change associated with each individual process. That is why so far only the total (summed) amplitude of the first decays was used to calculate the total free energy change associated with the $S_{4}$ intermediate formation (as shown in Figure 2), exploiting the relative independence of the sum of the individual amplitudes (pre-exponential factors) on the simulation approach (Buchta et al., 2007; Zaharieva et al., 2013).

To obtain in a more reliable way the time constants of all intermediate steps, a joint-fit approach for simulation of set of $D F$ decays measured at different temperatures was proposed in Zaharieva et al. (2013): DF decays measured at 7 different temperatures (same data set used also in the present study) were simulated implementing the Arrhenius equation to interrelate the rate constants at different temperatures:

$$
k_{i}=1 / \tau_{i}=k_{0_{i}} \exp \left(-E_{a_{i}} / k_{B} T\right) \quad \text { Eq. } 3.1
$$

In this way instead of four different rate constants for each of the 7 different temperatures, only 4 pre-exponential (frequency) factors $\left(k_{0_{i}}\right)$ and 4 activation energies $\left(E_{a_{i}}\right)$ were used. These 8 parameters are the same for all temperatures, assuming linear Arrhenius plots in the temperature range from 0 to $30^{\circ} \mathrm{C}$. The linear Arrhenius plot is predicted by standard electron-transfer as well as transition-state theory and largely has been experimentally confirmed for the reactions in the oxygen-evolving complex of PSII (Klauss et al., 2012; Bao and Burnap, 2015; Klauss et al., 2015; Gates et al., 2016). Using this rationale, instead of $7 \times 9=63$ adjustable parameter for the set of $7 \mathrm{DF}$ decays measured at different temperatures, the overall number of parameters was reduced to $8+5 \times 7=43$ free parameters (Zaharieva et al., 2013).

Although this approach allowed for more reliable determination of the rate constants, the precise determination of 
the amplitudes and thus the change in the free energy associated to the individual intermediates was not possible. Instead, only the total change in Gibbs free energy was determined using the sum of the individual amplitudes, as shown in Figure 2. In this study, we approach the thermodynamic parameters of the individual transitions implementing another restrain to the joint-fit simulation:

$$
\Delta G_{i}=\Delta H_{i}-T \Delta S_{i}
$$

Using an analogy to Eq. 1.2 and the approach presented in Figure 2, the change of the Gibbs free energy for the individual reaction steps can be expressed as:

$$
\begin{aligned}
\Delta G_{4} & =-k_{B} T \ln \frac{a_{4}+c}{c} \\
\Delta G_{3} & =-k_{B} T \ln \frac{a_{3}+a_{4}+c}{a_{4}+c} \\
\Delta G_{2} & =-k_{B} T \ln \frac{a_{2}+a_{3}+a_{4}+c}{a_{3}+a_{4}+c} \\
\Delta G_{1} & =-k_{B} T \ln \frac{a_{1}+a_{2}+a_{3}+a_{4}+c}{a_{2}+a_{3}+a_{4}+c}
\end{aligned}
$$

where $a_{i}$ denote the amplitudes of the individual kinetic components and $c$ is constant (see Eq. 2.1). This allows us to calculate the amplitude of each component, $a_{i}$, from $\Delta G_{i}$ and the amplitudes of the rest of the kinetic components. Eq. 3.2 allows us to calculate the individual amplitudes as function of the changes in enthalpy and entropy during the reaction using the set of Eqs. 3.3-3.6. In this way the constant from Eq. 2.1 remains the only parameter to be determined by free variation during the simulation. In addition, changes in the enthalpy, $\Delta H$, and entropy, $\Delta S$, associated to each transition are explicitly added to the model (but they are independent on the temperature). For the entire temperature set of 7 decays considered in the joint-fit approach this results in replacement of $5 \times 7=35$ amplitudes by 7 constants and 2 thermodynamics parameters for each of the 4 kinetic components, or 15 independent parameters. The total number of free parameters for the set of $7 \mathrm{DF}$ decays measured at 7 different temperatures thus decreases to 23 [ 4 frequency factors, 4 activation energies, 4 enthalpies, 4 entropic contributions and 7 constants (offsets)].

Using the simulation approach outlined here, the average number of free parameters used for each single decay decreases from 9 in the free 4 exponentials fit to $23 / 7=3.28$, or it approaches the number of the free parameters for the case of single exponential decay. A comparison of the residuals from the fit using different number of exponentials and the joint-fit model is shown in the Supplementary Figure S1. We note that the measurements at different temperatures are independent, and fresh PSII sample was used for each of them.

\section{DF Decays After the Third Flash $\left(S_{3} \rightarrow S_{0}\right.$ $+\mathrm{O}_{2}$ Transition)}

The DF decays analyzed in this work were detected by applying a sequence of saturating ns-Laser flashes to dark adapted PSII

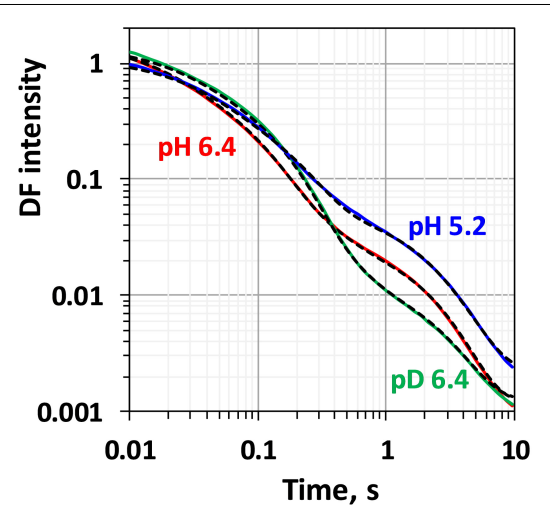

FIGURE 3 | Delayed fluorescence decays measured after the third flash at $20^{\circ} \mathrm{C}$ in $\mathrm{H}_{2} \mathrm{O}$ buffer with $\mathrm{pH} 6.4$ (red line), $\mathrm{pH} 5.2$ (blue line) and in $\mathrm{D}_{2} \mathrm{O}$ buffer with pD 6.4 (green line). Black dashed lines represent the simulated curves according to Eq. 2.1 within the joint fit simulation approach. Simulation parameters are shown in Table 1, Figures 4, 5 and

Supplementary Figure S3.

membrane particles and published in the Ph.D. thesis of Markus Grabolle (Grabolle, 2005). After the third Laser flash, the majority of PSII centers undergo $S_{3} \rightarrow S_{0}$ transition (Dau and Haumann, 2008). Delayed fluorescence decays measured after the third flash were simulated according to Eq. 2.1, where the slowest component represents the electron transfer step and $\mathrm{O}-\mathrm{O}$ bond formation (Figures 1, 2). This slow electron transfer step is well resolved and easily identified already by visual inspection as a plateau level in the $D F$ decay reached about $300 \mu \mathrm{s}$ after the flash (Figure 2B). It is now well established, that this process of $\mathrm{Mn}$ oxidation is preceded by proton removal from the OEC (Haumann et al., 2005; Dau and Haumann, 2007b; Klauss et al., 2012; Siegbahn, 2013). Previously it has been suggested that there could be three sequential reaction steps relating to proton removal form the OEC that occur before the onset of $\mathrm{Mn}$ reduction in the dioxygen-formation step (Dau and Haumann, $2007 b$ ). In order to model adequately the processes of proton and electron transfer occurring after the third flash in PSII, four exponentials are needed. The first three of them are related to the fast process of proton removal, but they are mathematically difficult to resolve. The very similar values obtained for the rate constants of these reactions when simulating the $D F$ decay measured at $20^{\circ} \mathrm{C}$ independently $\left(\mathrm{k}_{i}{ }^{-1}\right.$ equals to 17,72 , and $284 \mu \mathrm{s})$ and within the joint fit approach $\left(\mathrm{k}_{i}{ }^{-1}\right.$ equals to 15,65 , and $208 \mu$ s, Supplementary Figure S1) confirm the validity of the model applied for the joint-fit analysis. Previously lifetimes of 14, 65 , and $203 \mu \mathrm{s}$ in free four-exponential fit of $D F$ decays measured under similar conditions were reported (Buchta et al., 2007).

Comparison between the decays measured at $20^{\circ} \mathrm{C}$ at $\mathrm{pH}$ 6.4 and 5.2 as well as in $\mathrm{H}_{2} \mathrm{O}$ and $\mathrm{D}_{2} \mathrm{O}$ is shown in Figure 3. (Full set of DF decays measured at all temperatures, including the simulated curves is shown in the Supplementary Figure S2; The confidence intervals of the fit parameters, as calculated from the covariance matrix of system, are shown in Supplementary Table S2). Time constants of the individual kinetic components (Figure 4) show that both $\mathrm{D}_{2} \mathrm{O}$ and a decrease of $\mathrm{pH}$ result in 


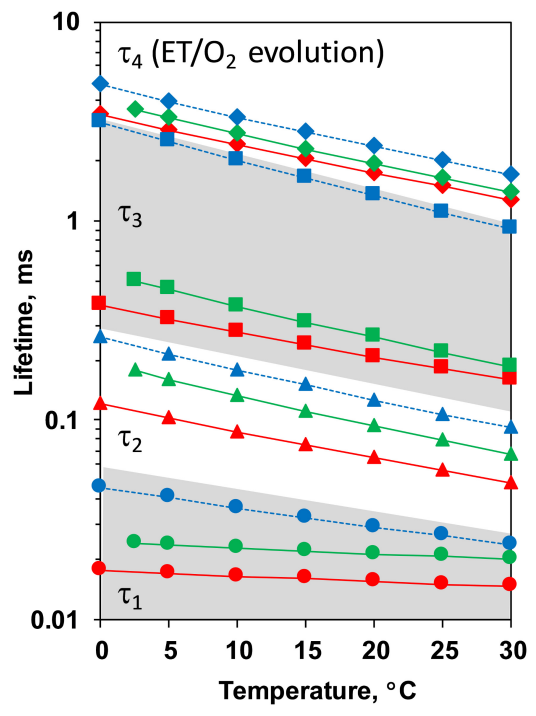

FIGURE 4 | Time constants of the DF decays measured after the third ns-Laser flash excitation of dark adapted PSII particles at pH 6.4 (red), pD 6.4 (green) and pH 5.2 (blue). The areas of the first and third kinetic components are shadowed in gray.

slowing down of all reactions. This effect is most drastic in the third kinetic component where the decrease of $\mathrm{pH}$ results in a change of $\tau_{3}$ from $208 \mu \mathrm{s}$ at $\mathrm{pH} 6.4$ to $1.33 \mathrm{~ms}$ at $\mathrm{pH} 5.2$ (values for $20^{\circ} \mathrm{C}$, see Table 1 ). The least affected from isotope exchange or $\mathrm{pH}$ drop is the electron transfer step (the slowest reaction).

The slope of the temperature dependence of the time constants remains almost unaffected for all components expect for the fastest one at $\mathrm{pH} 5.2$ (Figure 4). This indicates that there is no significant change in the activation energies, $E_{a}$, of the reactions. The total activation energy of the proton removal step, $E_{\text {arelax }}$, was $156 \mathrm{meV}$ at $\mathrm{pH} \quad 6.4$ and in qualitative agreement with the previously determined value of $180 \mathrm{meV}$ (Barry et al., 2006; Zaharieva et al., 2013).
$E_{\text {arelax }}$ at $\mathrm{pD} 6.4$ was similar $(184 \mathrm{meV})$ and increased to a value of $277 \mathrm{meV}$ at $\mathrm{pH} 5.2$ (Table 1). The activation energy of $234 \mathrm{meV}$ obtained for the dioxygen formation step (Table 1) agrees well with the previously determined value of $231 \mathrm{meV}$, using DF data (Buchta et al., 2007) as well as with the value of $230 \mathrm{meV}$, determined using photothermal beam deflection (PDB) data (Klauss et al., 2012) and time resolved absorption changes at $355 \mathrm{~nm}$ (Renger and Hanssum, 1992). However, for still unclear reasons, the $E_{a}$ determined here is significantly lower than previously determined activation energies of $340 \mathrm{meV}$ (near-UV data in Clausen et al., 2004), $420 \mathrm{meV}$ (polarographic data Clausen et al., 2004), and $380 \mathrm{meV}$ (dye-sensitized absorption measurements Haumann and Junge, 1994).

Kinetic isotope effect (KIE) for the H/D exchange was extensively studied before as KIE values higher than unity helps to identify the processes that involve proton movements. Values of 2.4 for the KIE for the $\mathrm{H}^{+}$removal step were obtained from optical absorption spectroscopy (Gerencser and Dau, 2010) and 2.5 from time-resolved X-ray spectroscopy (Zaharieva et al., 2016). For the O-O bond formation step lower values of 1.2 were determined by optical spectroscopy (Gerencser and Dau, 2010), 1.3 by photothermal beam deflection (Klauss et al., 2012) and about 1.4 by X-ray absorption spectroscopy (Zaharieva et al., 2016). Here, we obtain lower values of 1.6 for the $\mathrm{H}^{+}$removal step and 1.1 for the electron transfer step. We note that in order to facilitate the comparison to previous studies, instead of calculating the ratio between the rate constants of the individual kinetic components, we calculated the KIE of the $\mathrm{H}^{+}$release as a ratio between the mean lifetimes for all reactions before the oxygen formation step $\left[\tau_{\text {mean }}=\left(\tau_{1} \cdot a_{1}+\tau_{2} \cdot a_{2}+\tau_{3} \cdot a_{3}\right) /\left(a_{1}+a_{2}\right.\right.$ $\left.a_{3}\right)$ ]. Although it is not clear if these systematically lower values are due to the specifics of the $D F$ measurements or are imposed by the analysis, the trend of observing higher KIE for the proton release step is confirmed.

The temperature dependence of the Gibbs free energy, $\Delta G$, is shown in Figure 5. Notably, both the isotope exchange and $\mathrm{pH}$ decrease have a strong effect on $\Delta G$ of the electron

TABLE 1 | Time constants $(\tau)$, activation energies $\left(E_{a}\right)$ and further thermodynamic parameters: Gibbs free energy $(\Delta G)$, enthalpy $(\Delta H)$ and the entropic contribution to the change in Gibbs free energy $(T \Delta S)$, as determined from simulations of the $D F$ after the third ns-Laser flash applied to dark-adapted PSII membrane particles.

\begin{tabular}{|c|c|c|c|c|c|c|c|}
\hline S-state transition & $\mathrm{S} 3 \rightarrow \mathrm{SO}$ & ET/PT & $\tau(\mu \mathrm{s})$ & $\mathrm{Ea}(\mathrm{meV})$ & $\Delta G(m e V)$ & $\Delta H(\mathrm{meV})$ & $-\mathrm{T} \Delta \mathrm{S}(\mathrm{meV})$ \\
\hline \multirow{2}{*}{$\mathrm{H}_{2} \mathrm{O}, \mathrm{pH} 6.4$} & & & 65 & 217 & -49 & 156 & -205 \\
\hline & $\mathrm{S}^{n} \rightarrow \mathrm{SO}^{+}$ & $\mathrm{e}^{-}\left(\mathrm{O}_{2}\right)$ & 1736 & 234 & -83 & 31 & -114 \\
\hline \multirow[t]{2}{*}{$\mathrm{D}_{2} \mathrm{O}, \mathrm{pD} 6.4$} & $\mathrm{~S} 3^{+} \rightarrow \mathrm{S}^{n}$ & $\mathrm{H}^{+}$ & 21 & 47 & -16 & -91 & 74 \\
\hline & & & 93 & 253 & -59 & 189 & -248 \\
\hline \multirow[t]{4}{*}{$\mathrm{H}_{2} \mathrm{O}, \mathrm{pH} 5.2$} & $\mathrm{~S} 3^{+} \rightarrow \mathrm{S} 3^{n}$ & $\mathrm{H}^{+}$ & 29 & 158 & -21 & 2 & -23 \\
\hline & & & 127 & 249 & -53 & 44 & -97 \\
\hline & & & 1330 & 294 & -19 & -22 & 2 \\
\hline & $\mathrm{S}^{n} \rightarrow \mathrm{SO}^{+}$ & $\mathrm{e}^{-}\left(\mathrm{O}_{2}\right)$ & 2358 & 245 & -64 & -32 & -32 \\
\hline
\end{tabular}

For the time constants, $\Delta G$, and $T \Delta S$, the values for $20^{\circ} \mathrm{C}$ are presented. 

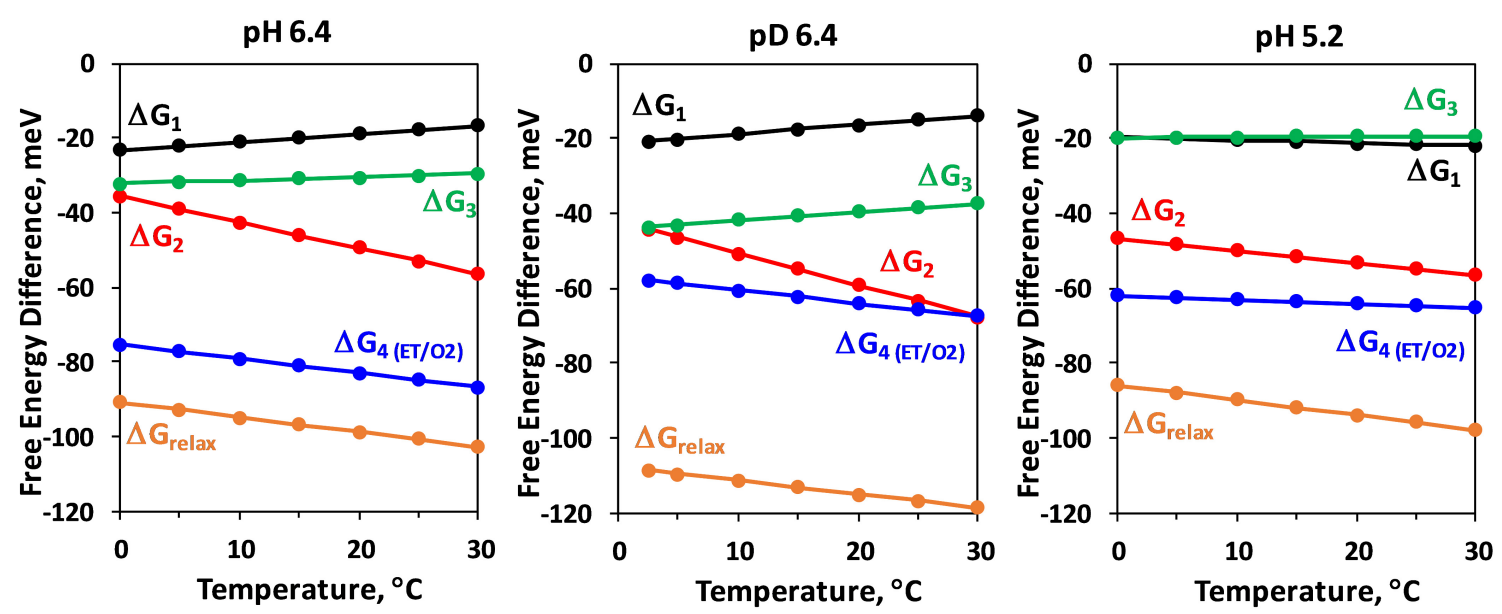

FIGURE 5 | Temperature dependence of the Gibbs energy, $\Delta G_{i}$, of the individual kinetic components resolved in the DF decay after the third Laser flash applied to dark adapted PSIl particles. $\Delta G_{\text {relax }}$, assignable to formation of a reaction intermediate after $\mathrm{Y}_{\mathrm{Z}}$ oxidation but before the electron transfer from $\mathrm{Mn}_{4} \mathrm{CaO}{ }_{5}$ complex and $\mathrm{O}_{2}$ evolution is also shown (for calculation of $\Delta G_{\text {relax }}$ see Figure 2).

transfer step $\left(\Delta G_{4}\right)$, significantly decreasing the change of $\Delta G$ as compared to the more natural control conditions $(\mathrm{pH}$ 6.4). Pronounced effect of $\mathrm{D}_{2} \mathrm{O}$ exchange and $\mathrm{pH}$ decrease on the free energy change of the reactions associated with $\mathrm{H}^{+}$release, $\Delta G_{\text {relax }}$, was reported earlier (Haumann et al., 2005), but on contrast to the electron transfer step, with $\mathrm{D}_{2} \mathrm{O}$ increasing the free energy drop, and low $\mathrm{pH}$ decreasing the change in $\Delta G_{\text {relax }}$. These results were reproduced in this study. Our results further show that these effects are mostly due to changes in the third kinetic component with lifetime of about $200 \mu$ s (Figure 5).

The negative slope of the temperature dependence of $\Delta G$ is related to the entropy increase during the reaction. This trend is observed for both, the $\mathrm{H}^{+}$release step $\left(\Delta G_{\text {relax }}\right)$ and for the

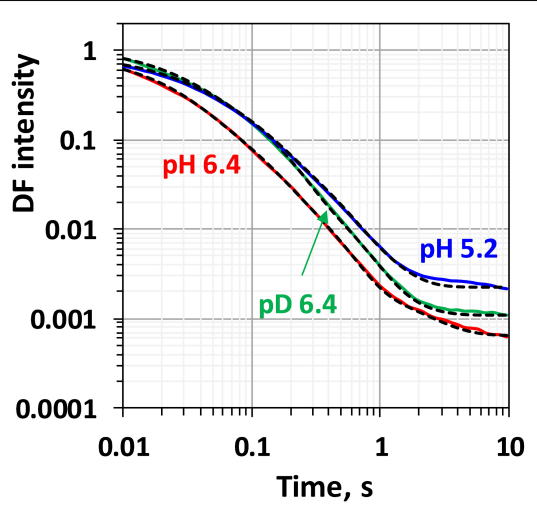

FIGURE 6 | Delayed fluorescence decays measured after the second flash at $20^{\circ} \mathrm{C}$ in $\mathrm{H}_{2} \mathrm{O}$ buffer with $\mathrm{pH} 6.4$ (red line), $\mathrm{pH} 5.2$ (blue line) and in $\mathrm{D}_{2} \mathrm{O}$ buffer with pD 6.4 (green line). Black dashed lines represent the simulated curves according to Eq. 2.1 within the joint fit simulation approach. Simulation parameters are shown in Table 2, Figures 7, 8 and Supplementary Figure S5. electron transfer step $\left(\Delta G_{4}\right)$ for all conditions. Figure 5 shows that while $\Delta G$ is always negative, some of the kinetic steps are characterized with positive slope of the temperature dependence and thus are associated with decrease of entropy. This is the case for first $(15 \mu \mathrm{s})$ and third $(200 \mu \mathrm{s})$ kinetic components at pL 6.4. Decrease of $\mathrm{pH}$ results in strong decrease of the entropic factor (Figure 5). The thermodynamic parameters obtained from the simulation are summarized in Table $\mathbf{1}$.

\section{DF Decays After the Second Flash $\left(S_{2} \rightarrow\right.$ $\mathrm{S}_{3}$ Transition)}

The second ns-Laser flash excitation applied to dark adapted PSII particles induces the $S_{2} \rightarrow S_{3}$ transition at the donor side of PSII (Dau and Haumann, 2008). DF decays recorded at $20^{\circ} \mathrm{C}$ after the second flash are shown in Figure 6 (DF decays measured at all temperatures, as well as for the simulated curves see Supplementary Figure S4; The confidence intervals of the fit parameters are shown in Supplementary Table S3). The $D F$ decays were simulated with sum of 4 exponential decays within the joint-fit approach as it was done for the DF decays recorded after the 3rd flash (Figure 2A). During the $S_{2} \rightarrow S_{3}$ transition proton is released form the $\mathrm{Mn}_{4} \mathrm{CaO}_{5}$ cluster, followed by electron transfer from the $\mathrm{Mn}_{4} \mathrm{CaO}_{5}$ complex to $\mathrm{Y}_{Z}^{\bullet+}$ (Dau et al., 2012). These processes were modeled by three exponents, assuming that the deprotonation is a bi-phasic process. The last component has very low amplitude (Figure 2A) and is likely related to processes in the acceptor side of the PSII, thus it is not of interest for this study. Although very good fit quality was achieved, in this case mixing of different components is more likely due to the absence of well resolved slow decay component. This affects the significance of the thermodynamic parameters determined by this approach as outlined in the following.

The time constants and activation energies determined here agree well with the literature values. The averaged life time of the deprotonation step preceding the electron transfer step, 
$\tau_{\text {mean }}=\left(\tau_{1} \cdot a_{1}+\tau_{2} \cdot a_{2}\right) /\left(a_{1}+a_{2}\right)$ at $20^{\circ} \mathrm{C}$ equals $29 \mu \mathrm{s}$ while the value determined by X-ray absorption spectroscopy was $26 \mu$ s (Zaharieva et al., 2016) and the value in Klauss et al. (2012) determined by photothermal beam deflection was $30 \mu \mathrm{s}$. The activation energy of the reaction determined before using photothermal beam deflection was $470 \mathrm{meV}$ (Klauss et al., 2012) while the activation energies of the two kinetic components related to the deprotonation step determined in this study are 49 and $397 \mathrm{meV}\left(E_{\text {arelax }}=271 \mathrm{meV}\right)$ (Table 2). For the electron transfer step we obtained time constant of $220 \mu$ s with $E_{a}=355 \mathrm{meV}$, in a relatively good agreement with the values reported previously of about $300 \mu$ s (Renger and Hanssum, 1992; Haumann et al., 1997; Haumann et al., 2005; Gerencser and Dau, 2010; Zaharieva et al., 2016) with $E_{a}=360 \mathrm{meV}$ (Renger and Hanssum, 1992; Haumann et al., 1997).

Despite the relatively good agreement between the rate constants and activation energies obtained here and reported in literature, there is a disagreement between our data and literature values for the kinetic isotope effect, $\mathrm{KIE}$, calculated as ratio between the rate constants in $\mathrm{H}_{2} \mathrm{O}$ and $\mathrm{D}_{2} \mathrm{O}$ buffers. With KIE for the proton release step of 1.5 (calculated as ratio between $\tau_{\text {mean }}$ ), we could not reproduce the large KIE of 4.5 (Zaharieva et al., 2016) or 5.6 reported earlier (Klauss et al., 2012). We also obtained KIE of 1.4 for the electron transfer step, a value smaller than the KIE of 1.7 reported before (Gerencser and Dau, 2010; Klauss et al., 2012) or 1.8 (Zaharieva et al., 2016). Still, in our calculations, although moderate in amplitude, the KIE is larger for the deprotonation step than for the electron-transfer step.

Another disagreement between the data presented in Table 2 and literature is related to the especially high entropic contribution for the deprotonation step (large temperature dependence of $\Delta G_{\text {relax }}$ ) reported earlier based on less restrictive joint-fit analysis of the same dataset (Zaharieva et al., 2013). These findings are at least partially explainable with the difficulties to simulate the rather featureless $D F$ decays measured after the second laser flash. Repeating the simulation with different starting values, we were able to get larger KIE of 1.6 for the proton release step and 2.2 for the electron transfer step

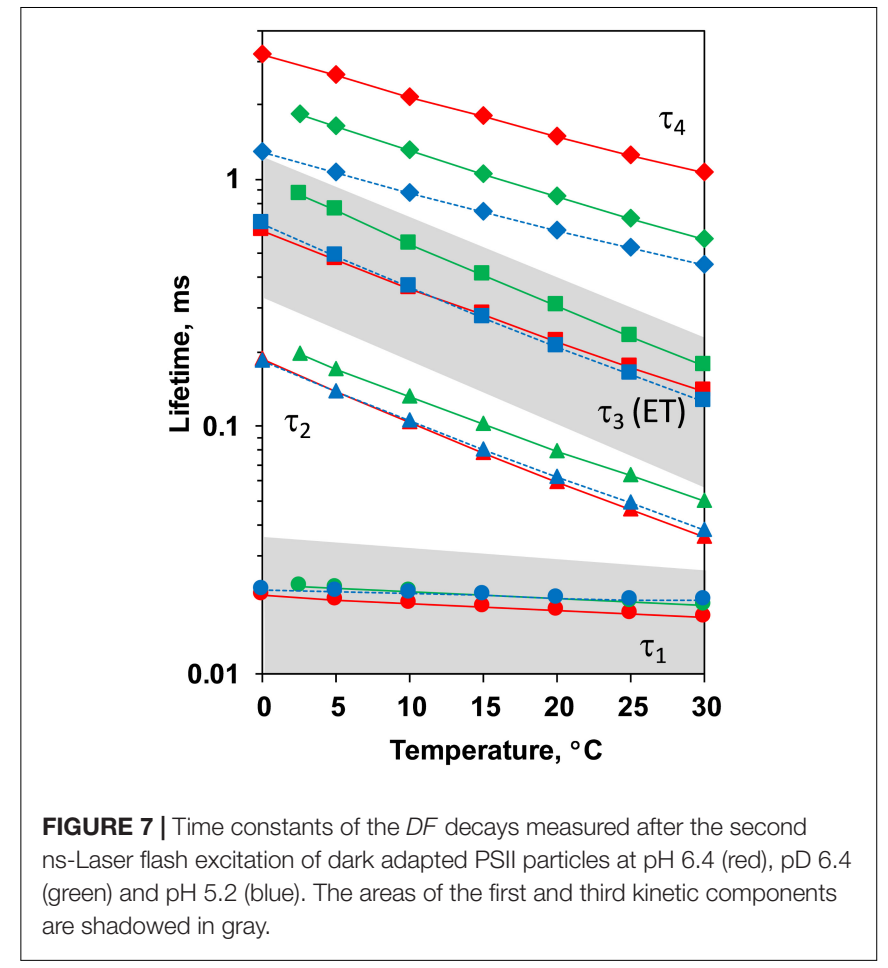

and to reproduce the steep temperature dependence of $\Delta G_{\text {relax }}$ with only slight decrease of the fit quality (see Supplementary Figures 6-9 and Supplementary Table 1). In this simulation however, as well as in the simulations in Zaharieva et al. (2013), there is disagreement with the literature values for the rate constants and activation energies as discussed above. These results suggests that even using highly restrictive simulation approach, there is still a large ambiguity in the results from simulation of the DF decays after the second flash and the thermodynamic parameters of the $\mathrm{S}_{2} \rightarrow \mathrm{S}_{3}$ transition determined by this approach should be discussed with care.

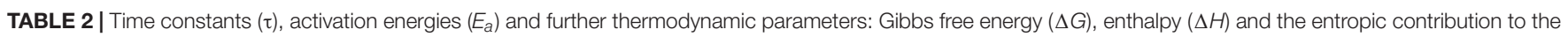
change in Gibbs free energy $(T \Delta S)$, as determined by simulations of the DF after the second ns-Laser flash applied to dark-adapted PSII membrane particles.

\begin{tabular}{|c|c|c|c|c|c|c|c|}
\hline S-state transition & $\mathrm{S} 2 \rightarrow \mathrm{S} 3$ & ET/PT & $\tau(\mu s)$ & $\mathrm{Ea}(\mathrm{meV})$ & $\Delta \mathrm{G}(\mathrm{meV})$ & $\Delta \mathbf{H}(\mathrm{meV})$ & $-\mathrm{T} \Delta \mathrm{S}(\mathrm{meV})$ \\
\hline \multirow[t]{4}{*}{$\mathrm{H}_{2} \mathrm{O}, \mathrm{pH} 6.4$} & $\mathrm{~S}^{+} \rightarrow \mathrm{S} 2^{n}$ & $\mathrm{H}^{+}$ & 18 & 49 & -30 & 10 & -40 \\
\hline & & & 60 & 393 & -44 & -102 & 59 \\
\hline & $\mathrm{S} 2^{n} \rightarrow \mathrm{S}^{+}$ & $e^{-}$ & 220 & 355 & -74 & -121 & 47 \\
\hline & & & 1490 & 262 & -36 & -81 & 45 \\
\hline \multirow[t]{4}{*}{$\mathrm{D}_{2} \mathrm{O}, \mathrm{pD} 6.4$} & $\mathrm{~S}^{+} \rightarrow \mathrm{S}^{n}$ & $\mathrm{H}^{+}$ & 20 & 47 & -22 & -15 & -7 \\
\hline & & & 79 & 356 & -57 & -20 & -37 \\
\hline & $\mathrm{S}^{n} \rightarrow \mathrm{S}^{+}$ & $e^{-}$ & 304 & 417 & -57 & -103 & 46 \\
\hline & & & 850 & 305 & -38 & -219 & 181 \\
\hline \multirow[t]{4}{*}{$\mathrm{H}_{2} \mathrm{O}, \mathrm{pH} 5.2$} & $\mathrm{~S}^{+} \rightarrow \mathrm{S}^{n}$ & $\mathrm{H}^{+}$ & 20 & 23 & -15 & -32 & 17 \\
\hline & & & 62 & 374 & -37 & -122 & 85 \\
\hline & $\mathrm{S}^{n}{ }^{n} \rightarrow \mathrm{S}^{+}$ & $e^{-}$ & 209 & 393 & -48 & -62 & 15 \\
\hline & & & 618 & 249 & -52 & -137 & 84 \\
\hline
\end{tabular}

For the time constants, $\Delta G$, and $T \Delta S$, the values for $20^{\circ} \mathrm{C}$ are presented. 


\section{DISCUSSION}

The extended S-state cycle (Figure 1) extends Kok's classical reaction cycle by considering explicitly the removal of protons from the Mn-complex (Dau and Haumann, 2007a,b). The proton removal step that precedes the electron transfer in the $S_{3} \rightarrow S_{0}$ transition was concluded from time-resolved XAS experiments in 2005, when a $200 \mu \mathrm{s}$ lag-phase (delay) before onset of the $\mathrm{Mn}$ reduction that is coupled to $\mathrm{O}_{2}$ formation was observed (Haumann et al., 2005). In conjunction with DF data reported in the same study and further results (Rappaport et al., 1994; Haumann et al., 2008; Gerencser and Dau, 2010; Rappaport et al., 2011; Klauss et al., 2012, 2015), the time-resolved XAS experiment provided conclusive evidence for alternating proton and electron removal from the $\mathrm{Mn}_{4} \mathrm{CaO}_{5}$ cluster before O-O bond formation, with proton removal preceding the electron transfer step. Experimental evidences for the temporal sequence of events during the $S_{2} \rightarrow S_{3}$ transition as proposed by the extended S-cycle model were obtained in 2012 by tracking of the proton-removal step in time-resolved photothermal beam deflection experiments (Klauss et al., 2012, 2015) and in recent time-resolved XAS experiments (Zaharieva et al., 2016). Here, we obtained similar rate constants for both, proton removal and electron transfer steps as determined before. Good agreement was found also with respect to the activation energies of the proton and electron transfer steps, as determined from the temperature dependence of the rate constants (see "Results" section).

Delayed fluorescence decays after saturating ns-Laser-flash excitation allow to go a step further and to resolve additional kinetic components that are currently not accessible by other experimental methods. To do that, a more comprehensive method for analysis, going beyond the simple three-exponential fit is needed and such approach is presented in the present study. Moreover, DF decays allow for direct estimation of the ratio between equilibrium populations of the states (equivalent to equilibrium constant) and thus to determine directly the differences in the Gibbs free energy, $\Delta G$, as detailed by Eqs. 1.1 and 1.2 and as illustrated by Figures $\mathbf{1 B}, \mathbf{2}$. The rationale has been described in detail also in Grabolle and Dau (2005) and Buchta et al. (2007). The temperature dependence of $\Delta G$ provides access to the entropic and enthalpic contribution to the total change in Gibbs free energy change, according to the equation 3.2. We note that the values for energetic parameters are not directly related to the activation entropy and activation enthalpy as they can be obtained with the Eyring equation from the temperature dependence of experimentally determined rate constants.

A deprotonation process is predicted to be associated with a sizable or even dominating entropic contribution to the free energy change ( $T \Delta S>\Delta H$, entropically driven reaction) and positive $\Delta H$ (endergonic process), due to the entropy increase associated with the "dilution" of the proton released in the bulk water. This is observed for the total change in Gibbs free energy during the $\mathrm{Y}_{\mathrm{Z}}{ }^{\bullet} S_{3}{ }^{+} \rightarrow \mathrm{Y}_{\mathrm{Z}}{ }^{\bullet} S_{3}{ }^{n}$ transition ( $\Delta G_{\text {relax }}$ in Figure 5). As expected, lowering $\mathrm{pH}$ (increase of proton concentration) decreases the absolute value of free energy drop associated with the deprotonation, while the $\mathrm{H} / \mathrm{D}$ isotope exchange has an opposite effect. These results for $\Delta G_{\text {relax }}$ during $S_{3} \rightarrow S_{4}$ transition were reported previously (Haumann et al., 2005), but our approach allows for individual analysis of the three kinetic components associated with the deprotonation step. The negative slope in the temperature dependence of $\Delta G_{\text {relax }}$ is mirrored by the $65 \mu \mathrm{s}$ component $\left(\Delta G_{2}\right)$, but surprisingly $\Delta G_{\text {relax }}$ of the second kinetic component shows almost no dependence on $\mathrm{pH}$ or H/D isotope exchange (Figure 5). The $\mathrm{pH}$ and $\mathrm{H} / \mathrm{D}$ dependence is reproduced by the third kinetic component with $200 \mu$ s lifetime $\left(\Delta G_{3}\right.$, Figure 5). A possible interpretation of these results is that during the $S_{3} \rightarrow S_{0}$ transition the proton release into the aqueous bulk occurs with $65 \mu \mathrm{s}$, preceded by $(15 \mu \mathrm{s})$ rearrangement of a protein-internal H-bonded proteinwater network and followed by $(200 \mu \mathrm{s})$ protein-internal proton relocation from the catalytic core of the OEC toward a nearby site. The slow process is delayed with decrease of $\mathrm{pH}$ where
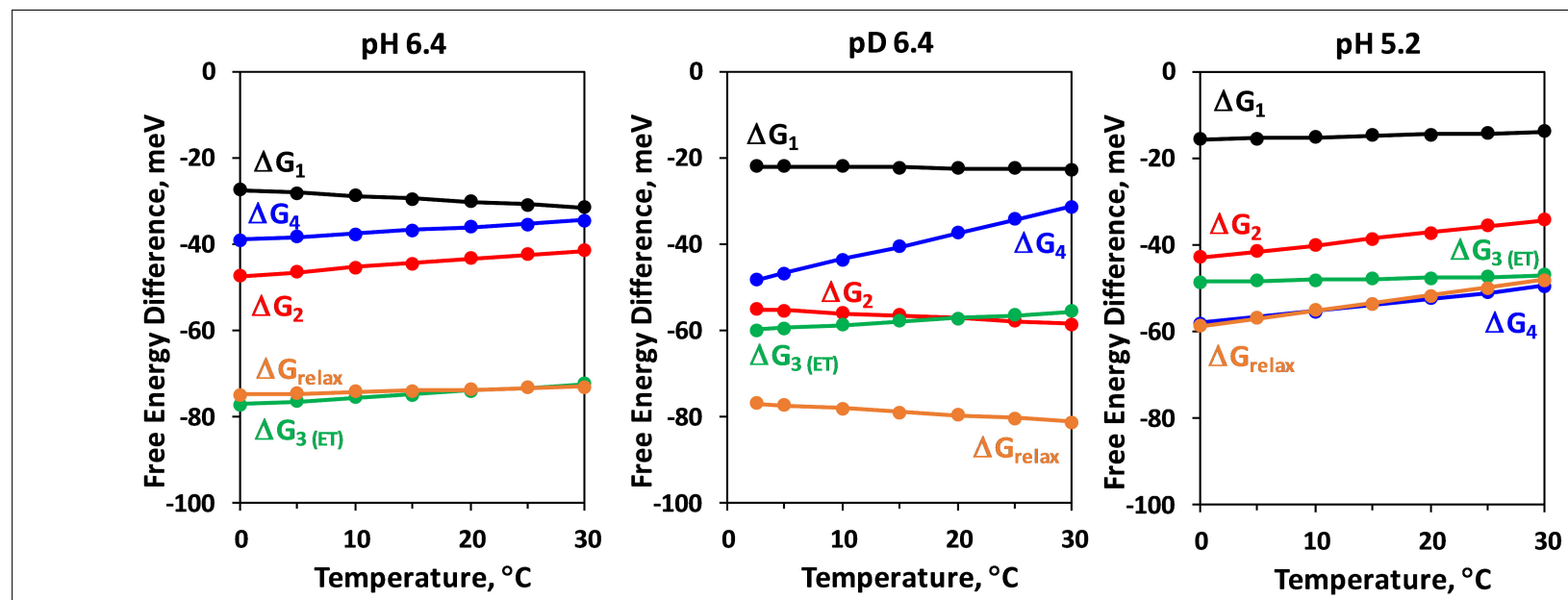

FIGURE 8 | Temperature dependence of the Gibbs energy, $\Delta G_{i}$, of the individual kinetic components resolved in the $D F$ decay after the second ns-Laser flash applied to dark adapted PSIl particles. $\Delta G_{\text {relax }}$, assignable to formation of a reaction intermediate after $\mathrm{Y}_{Z}$ oxidation but before the electron transfer from $\mathrm{Mn}_{4} \mathrm{CaO}_{5}$ complex is also shown (for calculation of $\Delta G_{\text {relax }}$ see Figure 2). 
the higher degree of protonation hampers the proton relocation within the protein-water matrix (increase of $\tau_{3}$ from $200 \mu \mathrm{s}$ at $\mathrm{pH} 6.4$ to $1.3 \mathrm{~ms}$ at $\mathrm{pH} 5.2$, see Table 1). The proton relocation could include proton movements from (substrate) water species bound at the $\mathrm{Mn}_{4} \mathrm{CaO}_{5}$ core of the OEC toward amino acid groups close to $\mathrm{Mn}_{4} \mathrm{CaO}_{5}$ complex. It also may involve proton relocation along proton channels extending toward carboxylate clusters at the protein surface (Haumann and Junge, 1994; Dau and Haumann, 2008; Bondar and Dau, 2012; Karge et al., 2014). The relatively small activation energy, the enthalpy decrease and the small dependence on the $\mathrm{pH}$ and isotope exchange of the fastest $15 \mu \mathrm{s}$ kinetic component implies that it may be related to fast reorganizations e.g., proton movements within hydrogen bonds induced through bond or through space (electric fields) interactions with the photooxidized $\mathrm{Y}_{Z}{ }^{\bullet+}$ (Styring et al., 2012; Nakamura et al., 2014). The sequence of events thus could be the following:

(1) Light-induced formation of $\mathrm{Y}_{\mathrm{Z}}{ }^{\bullet+}$ is followed by an initial rearrangement of the $\mathrm{H}$-bonded protein-water cluster surrounding the catalytic metal cluster $(15 \mu \mathrm{s})$.

(2) Long-range electrostatic interactions of the positively charged $\mathrm{Y}_{\mathrm{Z}}^{\bullet+}$ protonated peripheral groups (most likely carboxylate sidechains) result in deprotonation and proton release into the aqueous bulk, possibly (or even likely) followed immediately by proton transfer from the "outskirts" of the OEC toward the previously deprotonated group at the periphery of the PSII protein complex.

(3) A proton is relocated from the core of the metal cluster $\left(\mathrm{Mn}_{4} \mathrm{CaO}_{5}\right.$ core plus first-sphere water ligands) toward the OEC outskirts $(200 \mu \mathrm{s})$. Completion of this deprotonation step represents the pre-requisite of subsequent electron transfer and $\mathrm{O}-\mathrm{O}$ bond formation.

(4) An electron is transferred from the Mn ions of the OEC (Mn oxidation) or directly from a "substrate oxygen" (ligand oxidation) followed immediately by $\mathrm{O}-\mathrm{O}$ bond formation associated with $\mathrm{Mn}$ reduction and eventually $\mathrm{O}_{2}$ release $(1.7 \mathrm{~ms})$.

The interpretation of the results for the second laser flash $\left(S_{2} \rightarrow S_{3}\right)$ is less straightforward, also because of the ambiguity of the simulation results. In this case we use only two kinetic components to simulate the deprotonation step which precedes the electron transfer from $\mathrm{Mn}_{4} \mathrm{CaO}_{5}$ cluster to $\mathrm{Y}_{\mathrm{Z}}{ }^{\bullet+}$. A clear similarity is observed between the first kinetic component resolved in the DF decay after the second and the third flash, in terms of time constant (about $15 \mu \mathrm{s}$ ), surprisingly low activation energy, relative independence on $\mathrm{pH}$ and isotope exchange as well as low entropic contribution to the free energy drop (Table 2 and Figure 8). These similarities suggest that in both $S_{2} \rightarrow S_{3}$ transition and $S_{3} \rightarrow S_{0}$ transition, the deprotonation step is initiated by similar rearrangements in the proton bonding network possibly induced by the nearby $\mathrm{Y}_{\mathrm{Z}}{ }^{\bullet+}$. The likely deprotonation step in the $\mathrm{Y}_{\mathrm{Z}}{ }^{\bullet} S_{2}{ }^{+} \rightarrow \mathrm{Y}_{\mathrm{Z}} \bullet S_{2}{ }^{\mathrm{n}}$ transition (second kinetic component) has similar time constant of about $65 \mu \mathrm{s}$ as the one found in $\mathrm{Y}_{\mathrm{Z}}{ }^{\bullet} S_{3}{ }^{+} \rightarrow \mathrm{Y}_{\mathrm{Z}}{ }^{\bullet} S_{3}{ }^{\mathrm{n}}$ transition, but is characterized by a larger activation energy; and it is not an entropically driven reaction (according to Figure $\mathbf{8}$ and Table $\mathbf{2}$ there is even a slight decrease in entropy observed in both $\mathrm{pH}$ 6.4 and $\mathrm{pH}$ 5.2). By the latter characteristics as well as by the strong $\mathrm{pH}$ dependence this component is rather similar to the third component resolved in $S_{3} \rightarrow S_{0}$ transition (tentatively assigned to reorganization of $\mathrm{H}$-bonding network and decrease of total energy during $\mathrm{YZ}_{\mathrm{Z}}{ } S_{3}{ }^{+} \rightarrow \mathrm{Y}_{\mathrm{Z}}{ }^{\bullet} S_{3}{ }^{\mathrm{n}}$ the transition). For the electron transfer in the $S_{2} \rightarrow S_{3}$ we detect values of 200$300 \mu \mathrm{s}$, which is slightly faster, but still reasonably close to the figures determined by other methods (Renger and Hanssum, 1992; Haumann et al., 1997; Haumann et al., 2005; Gerencser and Dau, 2010; Zaharieva et al., 2016). Similarly, also the activation energy agrees reasonably well with previously published figures (Renger and Hanssum, 1992; Haumann et al., 1997). However, in clear contrast to other methods that detect the rate constant of electron transfer more specifically, we do not observe any slowing down at lower $\mathrm{pH}$. Moreover, a fourth exponential component of unclear origin is required for high-quality simulations, with time constants at $20^{\circ} \mathrm{C}$ of $1.5 \mathrm{~ms}\left(\mathrm{pH} 6.4, \mathrm{H}_{2} \mathrm{O}\right), 850 \mu \mathrm{s}\left(\mathrm{pD} 6.4, \mathrm{D}_{2} \mathrm{O}\right)$

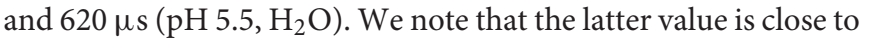
the time constant determined for the electron transfer step in the $S_{2} \rightarrow S_{3}$ transition (at $20^{\circ} \mathrm{C}$ and $\mathrm{pH} 5.5$ ) by UV-vis spectroscopy (Gerencser and Dau, 2010). This fourth component might relate to acceptor side processes, but some aspects like the acceleration in $\mathrm{D}_{2} \mathrm{O}$ cannot be explained easily, neither by a donor side nor an acceptor side process. Taking into account these discrepancies, we conclude that most likely the used simulation approach failed to describe the events in the $S_{2} \rightarrow S_{3}$ transition appropriately.

\section{DATA AVAILABILITY}

All datasets generated for this study are included in the manuscript and/or the Supplementary Files.

\section{AUTHOR CONTRIBUTIONS}

HD initiated the study. IZ and HD designed the model and wrote the manuscript. IZ performed the simulations.

\section{FUNDING}

Financial support from the Collaborative Research Center on Protonation Dynamics in Protein Function (CRC 1078, Project A4) is gratefully acknowledged.

\section{ACKNOWLEDGMENTS}

We thank Dr. Petko Chernev for supporting the simulations.

\section{SUPPLEMENTARY MATERIAL}

The Supplementary Material for this article can be found online at: https://www.frontiersin.org/articles/10.3389/fpls.2019.00386/ full\#supplementary-material 


\section{REFERENCES}

Bao, H., and Burnap, R. L. (2015). Structural rearrangements preceding dioxygen formation by the water oxidation complex of photosystem II. Proc. Natl. Acad. Sci. U.S.A. 112, E6139-E6147. doi: 10.1073/pnas.1512008112

Barry, B. A., Cooper, I. B., De Riso, A., Brewer, S. H., Vu, D. M., and Dyer, R. B. (2006). Time-resolved vibrational spectroscopy detects protein-based intermediates in the photosynthetic oxygen-evolving cycle. Proc. Natl. Acad. Sci. U.S.A. 103, 7288-7291. doi: 10.1073/pnas.0600216103

Blankenship, R. E. (2002). Molecular Mechanisms of Photosynthesis. Oxford: Blackwell Science. doi: 10.1002/9780470758472

Bondar, A. N., and Dau, H. (2012). Extended protein/water H-bond networks in photosynthetic water oxidation. Biochim. Biophys. Acta 1817, 1177-1190. doi: 10.1016/j.bbabio.2012.03.031

Buchta, J., Grabolle, M., and Dau, H. (2007). Photosynthetic dioxygen formation studied by time-resolved delayed fluorescence measurementsmethod, rationale, and results on the activation energy of dioxygen formation. Biochim. Biophys. Acta 1767, 565-574. doi: 10.1016/j.bbabio.2007.04.003

Chernev, P. (2007). Mathematical Approaches for Analysis of Photosystem 2 Chlorophyll Fluorescence. Ph.D. Thesis. Sofia: Department of Biology, Sofia University "St. Kliment Ohridski”.

Clausen, J., Debus, R. J., and Junge, W. (2004). Time-resolved oxygen production by PSII: chasing chemical intermediates. Biochim. Biophys. Acta. 1655, 184-194. doi: 10.1016/j.bbabio.2003.06.001

Dau, H. (1994). Molecular mechanisms and quantitative models of variable photosystem II fluorescence. Photochem. Photobiol. 60, 1-23. doi: 10.1111/j. 1751- 1097.1994.tb03937.x

Dau, H., and Haumann, M. (2007a). Eight steps preceding O-O bond formation in oxygenic photosynthesis-a basic reaction cycle of the photosystem II manganese complex. Biochim. Biophys. Acta 1767, 472-483. doi: 10.1016/j. bbabio.2007.02.022

Dau, H., and Haumann, M. (2007b). Time-resolved X-ray spectroscopy leads to an extension of the classical S-state cycle model of photosynthetic oxygen evolution. Photosynth. Res. 92, 327-343. doi: 10.1007/s11120-007-9141-9

Dau, H., and Haumann, M. (2008). The manganese complex of photosystem II in its reaction cycle-basic framework and possible realization at the atomic level. Coord. Chem. Rev. 252, 273-295. doi: 10.1016/j.ccr.2007.09.001

Dau, H., and Sauer, K. (1996). Exciton equilibration and photosystem II exciton dynamics - a fluorescence study on photosystem II membrane particles of spinach. Biochim. Biophys. Acta 1273, 175-190. doi: 10.1016/0005-2728(95) 00141-7

Dau, H., Zaharieva, I., and Haumann, M. (2012). Recent developments in research on water oxidation by photosystem II. Curr. Opin. Chem. Biol. 16, 3-10. doi: $10.1016 /$ j.cbpa.2012.02.011

Faller, P., Debus, R. J., Brettel, K., Sugiura, M., Rutherford, A. W., and Boussac, A. (2001). Rapid formation of the stable tyrosyl radical in photosystem II. Proc. Natl. Acad. Sci. U.S.A. 98, 14368-14373. doi: 10.1073/pnas.251382598

Faller, P., Goussias, C., Rutherford, A. W., and Un, S. (2003). Resolving intermediates in biological proton-coupled electron transfer: a tyrosyl radical prior to proton movement. Proc. Natl. Acad. Sci. U.S.A. 100, 8732-8735. doi: 10.1073/pnas. 1530926100

Ferreira, K. N., Iverson, T. M., Maghlaoui, K., Barber, J., and Iwata, S. (2004). Architecture of the photosynthetic oxygen-evolving center. Science 303, 1831-1838. doi: 10.1126/science.1093087

Gates, C., Ananyev, G., and Dismukes, G. C. (2016). The strontium inorganic mutant of the water oxidizing center (CaMn4O5) of PSII improves WOC efficiency but slows electron flux through the terminal acceptors. Biochim. Biophys. Acta 1857, 1550-1560. doi: 10.1016/j.bbabio.2016.06.004

Gerencser, L., and Dau, H. (2010). Water oxidation by photosystem II: H2O-D2O exchange and the influence of $\mathrm{pH}$ support formation of an intermediate by removal of a proton before dioxygen creation. Biochemistry 49, 10098-10106. doi: $10.1021 /$ bi101198n

Glasoe, P. K., and Long, F. A. (1960). Use of glass electrodes to measure acidities in deuterium oxide. J. Phys. Chem. 64, 188-190. doi: 10.1021/j100830a521

Goltsev, V., Zaharieva, I., Chernev, P., and Strasser, R. J. (2009). Delayed fluorescence in photosynthesis. Photosynth. Res. 101, 217-232. doi: 10.1007/ s11120-009-9451-1
Grabolle, M. (2005). Die Donorseite des Photosystems II: Rekombinationsfluoreszenz- und Röntgenabsorptionsstudien. Ph.D. Berlin: Fachbereich Physik, Freie Universität.

Grabolle, M., and Dau, H. (2005). Energetics of primary and secondary electron transfer in photosystem II membrane particles of spinach revisited on basis of recombination-fluorescence measurements. Biochim. Biophys. Acta 1708, 209-218. doi: 10.1016/j.bbabio.2005.03.007

Haumann, M., Bögershausen, O., Cherepanov, D., Ahlbrink, R., and Junge, W. (1997). Photosynthetic oxygen evolution: H/D isotope effects and the coupling between electron and proton transfer during the redox reactions at the oxidizing side of photosystem II. Photosynth. Res. 51, 193-208. doi: 10.1023/A: 1005861917596

Haumann, M., Grundmeier, A., Zaharieva, I., and Dau, H. (2008). Photosynthetic water oxidation at elevated dioxygen partial pressure monitored by timeresolved X-ray absorption measurements. Proc. Natl. Acad. Sci. U.S.A. 105, 17384-17389. doi: 10.1073/pnas.0802596105

Haumann, M., and Junge, W. (1994). Extent and rate of proton release by photosynthetic water oxidation in thylakoids: electrostatic relaxation versus chemical production. Biochemistry 33, 864-872. doi: 10.1021/bi00170a003

Haumann, M., Liebisch, P., Muller, C., Barra, M., Grabolle, M., and Dau, H. (2005). Photosynthetic $\mathrm{O} 2$ formation tracked by time-resolved $\mathrm{x}$-ray experiments. Science 310, 1019-1021. doi: 10.1126/science.1117551

Joliot, P., Barbieri, G., and Chabaud, R. (1969). Un nouveau modele des centres photochimiques du systeme II*. Photochem. Photobiol. 10, 309-329. doi: 10. 1111/j.1751-1097.1969.tb05696.x

Junge, W., Haumann, M., Ahlbrink, R., Mulkidjanian, A., and Clausen, J. (2002). Electrostatics and proton transfer in photosynthetic water oxidation. Philos. Trans. R. Soc. Lond. B 357, 1407-1418. doi: 10.1098/rstb.2002.1137

Kalaji, H. M., Schansker, G., Brestic, M., Bussotti, F., Calatayud, A., Ferroni, L., et al. (2017). Frequently asked questions about chlorophyll fluorescence, the sequel. Photosynth. Res. 132, 13-66. doi: 10.1007/s11120-016-0318-y

Kalaji, H. M., Schansker, G., Ladle, R. J., Goltsev, V., Bosa, K., Allakhverdiev, S. I., et al. (2014). Frequently asked questions about in vivo chlorophyll fluorescence: practical issues. Photosynth. Res. 122, 121-158. doi: 10.1007/s11120-014-0024-6

Karge, O., Bondar, A. N., and Dau, H. (2014). Cationic screening of charged surface groups (carboxylates) affects electron transfer steps in photosystem-II water oxidation and quinone reduction. Biochim. Biophys. Acta 1837, 1625-1634. doi: 10.1016/j.bbabio.2014.07.012

Kern, J., Chatterjee, R., Young, I. D., Fuller, F. D., Lassalle, L., Ibrahim, M., et al. (2018). Structures of the intermediates of Kok's photosynthetic water oxidation clock. Nature 563, 421-425. doi: 10.1038/s41586-018-0681-2

Klauss, A., Haumann, M., and Dau, H. (2012). Alternating electron and proton transfer steps in photosynthetic water oxidation. Proc. Natl. Acad. Sci. U.S.A. 109, 16035-16040. doi: 10.1073/pnas. 1206266109

Klauss, A., Haumann, M., and Dau, H. (2015). Seven steps of alternating electron and proton transfer in photosystem II water oxidation traced by time-resolved photothermal beam deflection at improved sensitivity. J. Phys. Chem. B 119, 2677-2689. doi: 10.1021/jp509069p

Kok, B., Forbush, B., and McGloin, M. (1970). Cooperation of charges in photosynthetic O2 evolution - I. A linear four-step mechanism. Photochem. Photobiol. 11, 457-475. doi: 10.1111/j.1751-1097.1970.tb06017.x

Lichtenthaler, H. K. (1987). Chlorophylls and carotenoids - pigments of photosynthetic biomembranes. Methods Enzymol. 148, 350-382. doi: 10.1016/ 0076-6879(87)48036- 1

Nakamura, S., Nagao, R., Takahashi, R., and Noguchi, T. (2014). Fourier transform infrared detection of a polarizable proton trapped between photooxidized tyrosine $\mathrm{Yz}$ and a coupled histidine in photosystem II: relevance to the proton transfer mechanism of water oxidation. Biochemistry 53, 3131-3144. doi: $10.1021 / \mathrm{bi500237y}$

Nelson, N., and Junge, W. (2015). Structure and energy transfer in photosystems of oxygenic photosynthesis. Ann. Rev. Biochem. 84, 659-683. doi: 10.1146/ annurev-biochem-092914-041942

Nelson, N., and Yocum, C. F. (2006). Structure and function of photosystems I and II. Ann. Rev. Plant Biol. 57, 521-567. doi: 10.1146/annurev.arplant.57.032905. 105350

Ort, D., and Yocum, C. F. (1996). Oxygenic Photosynthesis: The Light Reactions. Dordrecht: Kluwer Academic Publ. doi: 10.1007/0-306-48127-8 
Pagliano, C., Saracco, G., and Barber, J. (2013). Structural, functional and auxiliary proteins of photosystem II. Photosynth. Res. 116, 167-188. doi: 10.1007/s11120013-9803-8

Rappaport, F., Blanchard-Desce, M., and Lavergne, J. (1994). Kinetics of electron transfer and electrochromic change during the redox transition of the photosynthetic oxygen-evolving complex. Biochim. Biophys. Acta. 1184, 178-192. doi: 10.1016/0005-2728(94)90222-4

Rappaport, F., Ishida, N., Sugiura, M., and Boussac, A. (2011). Ca2+ determines the entropy changes associated with the formation of transition states during water oxidation by photosystem II. Energy Environ. Sci. 4, 2520-2524. doi: 10.1039/C1EE01408K

Renger, G., and Hanssum, B. (1992). Studies on the reaction coordinates of the water oxidase in PS II membrane fragments from spinach. FEBS Lett. 299, 28-32. doi: 10.1016/0014-5793(92)80092-U

Schatz, G. H., Brock, H., and Holzwarth, A. R. (1988). Kinetic and energetic model for the primary processes in photosystem II. Biophys. J. 54, 397-405. doi: 10.1016/S0006-3495(88)82973-4

Schiller, H., and Dau, H. (2000). Preparation protocols for high-activity photosystem II membrane particles of green algae and higher plants, $\mathrm{pH}$ dependence of oxygen evolution and comparison of the S2-state multiline signal by X-band EPR spectroscopy. J. Photochem. Photobiol. B 55, 138-144. doi: 10.1016/S1011-1344(00)00036-1

Siegbahn, P. E. M. (2013). Water oxidation mechanism in photosystem II, including oxidations, proton release pathways, $\mathrm{O}-\mathrm{O}$ bond formation and $\mathrm{O} 2$ release. Biochim. Biophys. Acta 1827, 1003-1019. doi: 10.1016/j.bbabio.2012. 10.006

Styring, S., Sjöholm, J., and Mamedov, F. (2012). Two tyrosines that changed the world: interfacing the oxidizing power of photochemistry to water splitting in photosystem II. Biochim. Biophys. Acta 1817, 76-87. doi: 10.1016/j.bbabio.2011. 03.016

Suga, M., Akita, F., Hirata, K., Ueno, G., Murakami, H., Nakajima, Y., et al. (2015). Native structure of photosystem II at 1.95 a resolution viewed by femtosecond X-ray pulses. Nature 517, 99-103. doi: 10.1038/nature13991

Umena, Y., Kawakami, K., Shen, J.-R., and Kamiya, N. (2011). Crystal structure of oxygen-evolving photosystem II at a resolution of $1.9 \AA$. Nature 473, 55-60. doi: 10.1038/nature09913 van Laarhoven, P. J. M., and Aarts, E. H. L. (1987). "Simulated annealing," in Simulated Annealing: Theory and Applications Mathematics and its Applications, eds P. J. M. van Laarhoven and E. H. L. Aarts (Dordrecht: Springer). doi: 10.1007/978-94-015-7744-1

Williamson, A., Conlan, B., Hillier, W., and Wydrzynski, T. (2011). The evolution of photosystem II: insights into the past and future. Photosynth. Res. 107, 71-86. doi: 10.1007/s11120-010-9559-3

Zaharieva, I., Dau, H., and Haumann, M. (2016). Sequential and coupled proton and electron transfer events in the S2 - > S3 transition of photosynthetic water oxidation revealed by time-resolved X-ray absorption spectroscopy. Biochemistry 55, 6996-7004. doi: 10.1021/acs.biochem. $6 \mathrm{~b} 01078$

Zaharieva, I., Grabolle, M., Chernev, P., and Dau, H. (2013). "Water oxidation in photosystem II: energetics and kinetics of intermediates formation in S2- > S3 and S3- > S0 transitions monitored by delayed chlorophyll fluorescence," in Photosynthesis Research for Food, Fuel and Future, eds T. Kuang, C. Lu, and L. Zhang (Heidelberg: Springer), 234-238.

Zaharieva, I., Wichmann, J. M., and Dau, H. (2011). Thermodynamic limitations of photosynthetic water oxidation at high proton concentrations. J. Biol. Chem. 286, 18222-18228. doi: 10.1074/jbc.M111.237941

Zouni, A., Witt, H. T., Kern, J., Fromme, P., Krauss, N., Saenger, W., et al. (2001). Crystal structure of photosystem II from Synechococcus elongatus at $3.8 \AA$ resolution. Nature 409, 739-743. doi: 10.1038/ 35055589

Conflict of Interest Statement: The authors declare that the research was conducted in the absence of any commercial or financial relationships that could be construed as a potential conflict of interest.

Copyright (c) 2019 Zaharieva and Dau. This is an open-access article distributed under the terms of the Creative Commons Attribution License (CC BY). The use, distribution or reproduction in other forums is permitted, provided the original author(s) and the copyright owner(s) are credited and that the original publication in this journal is cited, in accordance with accepted academic practice. No use, distribution or reproduction is permitted which does not comply with these terms. 